\title{
INCORPORAÇÃO DE NB EM ZEÓLITAS: USO COMO CATALISADORES NA DECOMPOSIÇÃO DO PERÓXIDO DE OXIGÊNIO
}

\author{
Selma Calgaroto, Cléber Calgaroto, Fábio G. Penha \\ Departamento de Química, Universidade Regional Integrada do Alto Uruguai e das Missões, Campus Erechim, Av. Sete de Setembro, \\ 1621, 99700-000 Erechim - RS. \\ Sibele B. C. Pergher* \\ Departamento de Química, Universidade Federal do Rio Grande do Norte, Av. Senador Salgado Filho, 3000, 59.078-970 Natal - RN. \\ Luiz Carlos A. Oliveira \\ Departamento de Química, Universidade Federal de Lavras, Caixa Postal 3037, CEP \\ 37200-000 Lavras-MG. \\ * e-mail: sibelepergher@gmail.com
}

\section{NIOBIUM INCORPORATION ON ZEOLITES: USE AS CATALYSTS IN THE $\mathrm{H}_{2} \mathrm{O}_{2}$ DECOMPOSITION.}

In this work, the comercial zeolites ZSM-5 and Y were submitted to previous ionic change with $\mathrm{NH}_{4} \mathrm{NO}_{3}$, followed by the addition of $\mathrm{Nb}$. The incorporation of $\mathrm{Nb}$ on its structure aims increasing the acidity and improved the catalyst activity of these zeolites. The materials obtained are characterized by X-Ray diffraction patterns, chemical analysis, SEM and textural analysis by $\mathrm{N}_{2}$ adsorption. Furthermore, the materials were avaliated by decomposition reaction of the $\mathrm{H}_{2} \mathrm{O}_{2}$. The $\mathrm{Nb}$ presence improved the

catalytic activity for $\mathrm{H}_{2} \mathrm{O}_{2}$ decomposition. Moreover, high amounts of $\mathrm{Nb}$ incorporated into zeolites caused a partial destruction of the structure of the materials as evidenced by

$\mathrm{X}$-Ray diffraction patterns.

Palavras-chave: ZSM-5; Y; Nb; decomposição catalítica.

\section{INTRODUÇÃO}

Os catalisadores contendo nióbio têm sido alvo de intensa pesquisa, principalmente devido à suas propriedades ácidas e redox [1-4]. Os compostos de nióbio exibem propriedades diferenciadas que não são observadas nos compostos de seus elementos vizinhos da tabela periódica. Algumas delas tais como a estabilidade ou a forte interação metal-suporte são muito importantes, dependendo da aplicação desejada [5]. Recentemente, o nióbio surgiu como um importante constituinte em catalisadores heterogêneos, agindo como componente cataliticamente ativo ou como promotor em formulações multicomponente, e estes catalisadores podem ser empregados em uma grande quantidade de reações de hidrogenação e oxidação[6]. Desta forma, uma alternativa interessante para se obter catalisadores com uma estrutura cristalina bem definida, com alta seletividade e com grande estabilidade térmica, seria a utilização de zeólitas[7] como matriz de estabilização.
Sendo assim, este trabalho tem por objetivo estudar a incoporação de $\mathrm{Nb}$ nas zeólitas ZSM-5 e Y visando aumentar a atividade destes materiais.

Zeólitas são aluminossilicatos hidratados, que apresentam um esqueleto cristalino formado pela combinação tridimensional de tetraedros $\mathrm{TO}_{4}(\mathrm{~T}=$ $\mathrm{Si}, \mathrm{Al}, \mathrm{Fe}, \mathrm{P} . .$.$) unidos entre si através de átomos de$ oxigênio. Nas zeólitas mais comuns, $\mathrm{T}$ representa aos elementos $\mathrm{Si}$ e Al. Sendo o alumínio trivalente, os tetraedros $\mathrm{AlO}_{4}$ induzem cargas negativas na estrutura, que são neutralizadas por cátions de compensação intercambiáveis. A estrutura das Zeólitas apresenta canais e cavidades de dimensões moleculares, nos quais se encontram moléculas de água, adsorbatos e cátions compensadores de carga.

A zeólita sintética do tipo Y tem em sua estrutura [7], a unidade fundamental sodalita, que é um octaedro truncado constituído de unidades tetraédricas de $\mathrm{AlO}_{4} \mathrm{e}$ $\mathrm{SiO}_{4}$. Estes octaedros truncados são interligados através 
de prismas hexagonais dando origem a vários tipos de cavidades. (Figura 1). A zeólita ZSM-5 caracteriza-se por um alto teor de silício ( $\mathrm{Si} / \mathrm{Al}>15)$. A cela unitária da ZSM-5 apresenta a seguinte fórmula empírica: $M_{n}$ $\mathrm{Al}_{\mathrm{n}} \mathrm{Si}_{(96-\mathrm{n})} \mathrm{O}_{192} \cdot 16 \mathrm{H}_{2} \mathrm{O}$, onde $\mathrm{M}$ é o cátion de valência $\mathrm{n}$. A ZSM-5 possui muitas aplicações industriais devida à sua alta seletividade em determinadas reações catalíticas e ao alto grau de estabilidade térmica e ácida [8]. Esta zeólita não possui cavidade. (Figura 2).

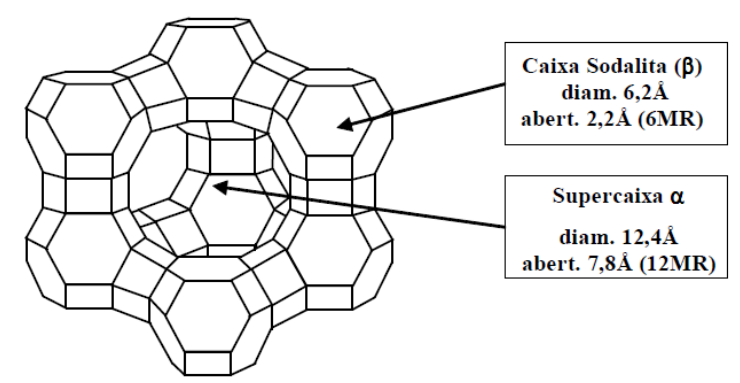

Figura 1. Estrutura da zeólita Y.

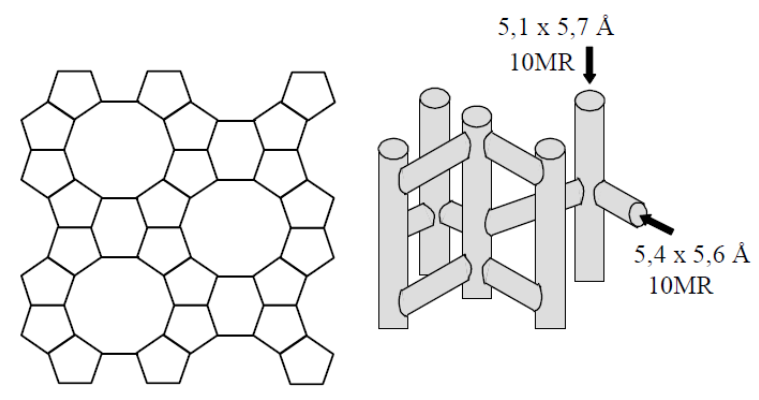

Figura 2. Estrutura da zeólita ZSM-5.

O Brasil é o maior produtor mundial de $\mathrm{Nb}$, representando $96,6 \%$ do total [9], o que torna economicamente viável o seu uso em diversos materiais. Atualmente, a aplicação mais importante desse metal é como elemento de liga [10]. Recentemente, diversos estudos têm relatado o uso de compostos de nióbio como catalisadores para a ativação de agentes oxidantes visando a oxidação de poluentes em meio aquoso [11-13]. Carvalho e colaboradores (2009) utilizaram composto de Nióbio em meio aquoso $\left(\mathrm{H}_{2} \mathrm{O}_{2}\right)$ e em fase gasosa $\left(\mathrm{O}_{2}\right)$ para degradação do corante azul de metileno. Em outro estudo [11], na degradação do mesmo corante, foram utilizados compostos de $\mathrm{Nb}$ associados com hematitas como catalisador em presença de $\mathrm{H}_{2} \mathrm{O}_{2}$ e luz UV. Assim, os catalisadores à base de nióbio são eficazes em processos de controle de poluição, oxidação seletiva, hidrogenação e desidrogenação, desidratação, hidratação, fotoquímica, eletroquímica e polimerização [14-15].
$\mathrm{OH}_{2} \mathrm{O}_{2}$ é um oxidante eficiente, seguro e de custo acessível, utilizado há algumas décadas em aplicações ambientais em todo o mundo [16]. A destruição de cloro residual e componentes reduzidos, tais como tiossulfato, sulfetos e sulfitos; redução da demanda química e bioquímica de oxigênio [17-19] - oxidação de poluentes orgânicos [20]; oxidação de componentes inorgânicos [21-22] são apenas alguns dos usos desse oxidante. O tratamento de águas e esgotos e efluentes indústrias empregando-se $\mathrm{H}_{2} \mathrm{O}_{2}$ é uma prática comum há pelo menos 20-25 anos em países desenvolvidos [23 $-25]$.

O peróxido de hidrogênio é relativamente estável em solução, sendo necessária uma espécie orgânica, um metal de transição ou radiação para ativálo. Esta ativação pode ser feita de diferentes maneiras: (i) reagindo-se $\mathrm{H}_{2} \mathrm{O}_{2}$ com espécies metálicas capazes de gerar espécies metal-peroxo ou metal-hidroperoxo, como Ti (IV), V(V), Mo (VI), Nb(V), W(VI) e Re (VII); (ii) usando $\mathrm{H}_{2} \mathrm{O}_{2}$ como doador de oxigênio, produzindo espécies metal-oxo, que são muito reativas. Nesses casos, utilizam-se sistemas baseados em Fe (III), Mn (II) e Ru (II). Nas últimas décadas, as espécies peroxo e hidroperoxo formadas com os metais de transição, Mo, $\mathrm{W}, \mathrm{V}, \mathrm{Nb}$, tem atraído especial atenção devido à sua coordenação química, importância biológica (atividade antitumoral), bem como seu papel como catalisador em diversos processos, como: oxidação de sulfetos, alquenos, álcoois, hidrocarbonetos e aromáticos e fosfinas [26].

\section{PARTE EXPERIMENTAL}

\section{Materiais}

As zeólitas ZSM-5 e Y utilizadas neste trabalho são zeólitas comerciais e foram fornecidas pelo Instituto de Tecnologia Química - ITQ de Valência. Os materiais preparados foram denominados conforme o tratamento recebido. Antes do nome da zeólita foi colocado o símbolo $\mathrm{H}$ para indicar que nestes materiais foi realizada uma troca iônica com $\mathrm{H}^{+}$. Após o nome da zeólita foi colocado o símbolo $\mathrm{Nb}$ para os materiais tratados com o composto de $\mathrm{Nb}$ e após este símbolo o número indicando a quantidade em gramas deste composto empregado no tratamento, exemplos: HZSM 5Nb0,5 e HYNb1.

\section{Troca iônica}

As zeólitas (ZSM-5 e Y) foram submetidas à 
troca iônica com $\mathrm{NH}_{4} \mathrm{NO}_{3} .12 \mathrm{~g}$ das zeólitas (ZSM-5 e Y) foram adicionadas a $180 \mathrm{~mL}$ de $\mathrm{NH}_{4} \mathrm{NO}_{3} 1 \mathrm{~mol} / \mathrm{L}$. A mistura permaneceu em refluxo a $80^{\circ} \mathrm{C}$ por 8 horas. Após este período, o sólido foi separado por filtração, lavado com água destilada e seco na estufa a $100^{\circ} \mathrm{C}$. As amostras foram calcinadas a $500^{\circ}$ por 5 horas, obtendose então as zeólitas (HZSM-5, HY).

\section{Zeólitas Trocadas com $\mathrm{Nb}^{*}$}

O composto de nióbio utilizado durante o trabalho foi o oxalato amoniacal de nióbio $\left(\mathrm{Nb}^{*}\right)$, cedido pela CBMM - Companhia Brasileira de Metalurgia e Mineração. Foram misturadas $2 \mathrm{~g}$ de zeólitas (HZSM5 , HY) em $40 \mathrm{~mL}$ de uma solução aquosa de oxalato amoniacal de nióbio contendo 0;5, 1; 5,0 g de $\mathrm{Nb}^{*}$. Em seguida aqueceu-se a mistura a $80^{\circ} \mathrm{C}$, deixando-a sob agitação durante 4 horas. Ao final desse tempo os sólidos são separados por filtração, lavados com água destilada e secos a $100^{\circ} \mathrm{C}$.

\section{Caracterização dos materiais}

Todos os materiais preparados foram caracterizados por diversas técnicas complementares: difração de raios-X, microscopia eletrônica de varredura, análise química e análises textural por adsorção de nitrogênio. A atividade catalítica foi avaliada através da reação de decomposição de $\mathrm{H}_{2} \mathrm{O}_{2}$.

\section{Difração de Raios-X}

As análises foram realizadas em laboratório de difratograma de raios-X no centro de estudos em petrologia e geoquímica - CPGq Instituto de geociências - da Universidade Federal do Rio Grande do Sul (UFRGS), sendo que o equipamento utilizado foi DIFFRAKTOMETER - modelo D5000 (Siemens) utilizando filtro de $\mathrm{Ni}$ e radiação $\mathrm{Cu}-\mathrm{Ka}(\lambda=1,54 \mathrm{~nm})$.

\section{Análise Química}

As amostras foram moídas em gral de ágata e decompostas em frasco de politetrafluoretileno com aquecimento por microondas sob pressão $\left(190^{\circ} \mathrm{C}\right)$ por $30 \mathrm{~min}$.

Foi usada a seguinte mistura ácida para a decomposição: $4 \mathrm{~mL}$ de $\mathrm{HNO}_{3}$, com $4 \mathrm{~mL}$ de $\mathrm{HF}$ concentrados. As determinações foram feitas na Universidade Federal de Santa Maria (UFSM), em um espectrômetro de emissão óptica com plasma indutivamente acoplado (ICP-OES), com nebuilizador do tipo GemCone, câmara de nebulização Scott, 309,418 nm, 269,706 e 292,781.

\section{Microscopia Eletrônica de Varredura}

As análises de microscopia eletrônica de varredura foram realizadas na Universidade Federal do Rio Grande do Sul (UFRGS), em um microscópio MEV SSZ 550 Shimadzu e as amostras foram previamente recobertas com um filme de ouro.

\section{Análise textural por adsorção de nitrogênio (Área $B E T)$}

As medidas de área superficial específica foram realizadas no Laboratório de Química Ambiental da URI - Erechim por adsorção de Nitrogênio, utilizando o aparelho QuantaChrome Nova 2200e. Para as análises se utiliza aproximadamente $100 \mathrm{mg}$ de amostra, e estas são tratadas a $150^{\circ} \mathrm{C}$ por aproximadamente 4 horas com fluxo de ar sintético, após este tratamento se realizam as medidas de adsorção/dessorção.

Teste Catalítico - Decomposição de peróxido de hidrogênio $\left(\mathrm{H}_{2} \mathrm{O}_{2}\right)$

A decomposição de $\mathrm{H}_{2} \mathrm{O}_{2}$, representada pela Equação 1, foi acompanhada pela medida volumétrica da quantidade de gás $\mathrm{O}_{2}$ formado, utilizando o sistema mostrado na Figura 3.

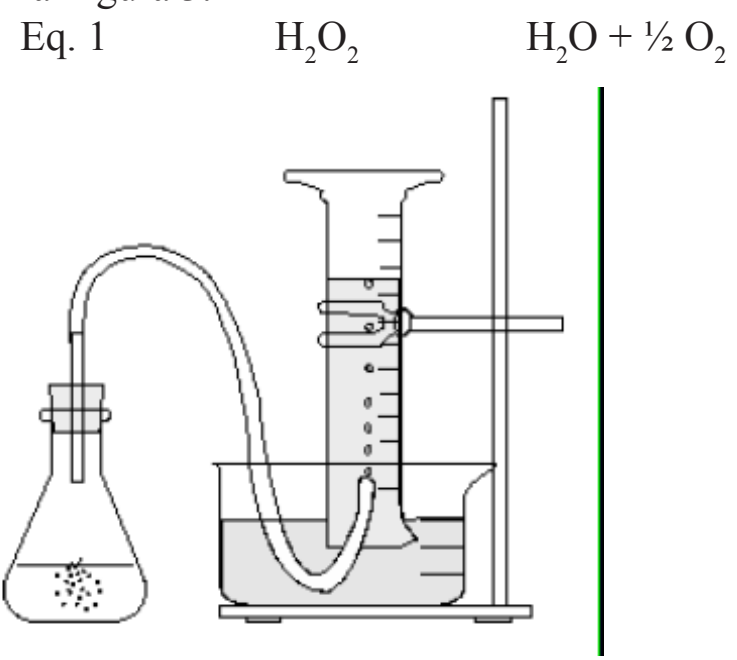

Figura 3. Sistema utilizado para estudar a decomposição de $\mathrm{H}_{2} \mathrm{O}_{2}$ catalisada pelos materiais com nióbio.

As reações foram realizadas a temperatura ambiente, utilizando-se $4 \mathrm{~mL}$ de solução de $\mathrm{H}_{2} \mathrm{O}_{2}(30 \%$ 
v/v), 5 mL de água mili-q e $100 \mathrm{mg}$ do material (zeólitas ZSM5 e Y). A mistura reacional foi mantida sob agitação magnética por aproximadamente 60 minutos. Anotaram-se os volumes de $\mathrm{O}_{2}$ liberado.

Testes catalíticos também foram realizados empregando a zeólita $\mathrm{HY} 0,5 \mathrm{Nb}$ e diferentes quantidades de catalisador $(50,100$ e $200 \mathrm{mg})$ e diferentes quantidades de $\mathrm{H}_{2} \mathrm{O}_{2}$. $(2,4$ e $8 \mathrm{~mL})$.

\section{RESULTADOS E DISCUSSÃO}

Os materiais foram inicialmente caracterizados por difratometria de raios-X. As Figuras 4 e 5 apresentam os difratogramas das amostras.

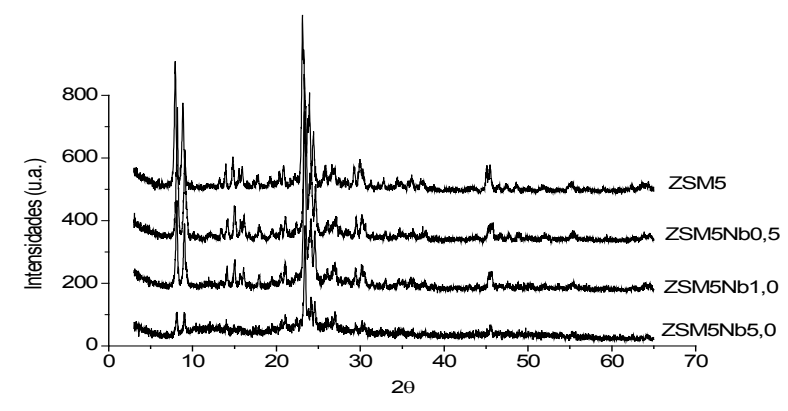

Figura 4. Difratograma de Raios X dos materiais obtidos da zeólita ZSM-5.

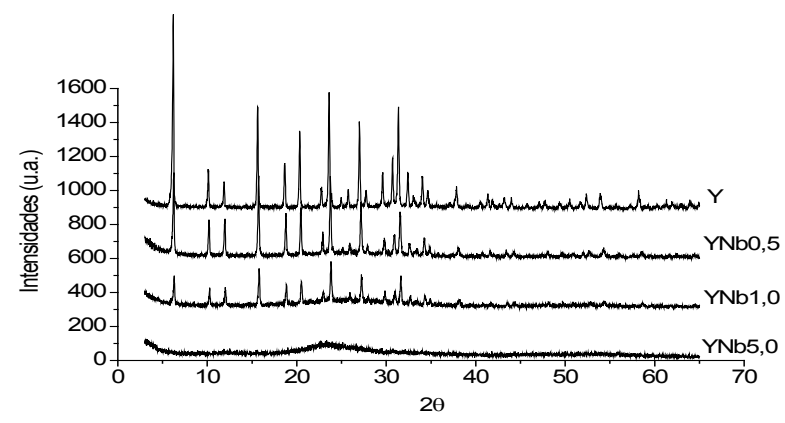

Figura 5. Difratograma de Raios $X$ dos materiais obtidos da zeólita Y.

Os resultados de difração de raios-X (Figuras 4 e 5), apontaram que maiores teores de $\mathrm{Nb}$ levam a uma destruição parcial da estrutura, este efeito é mais evidenciado na zeólita Y. A Tabela 1 apresenta as cristalinidades relativas dos materiais comprovando que a perda de cristalinidade da zeólita Yé muito elevada. Isso também é observado através das micrografias (Figura 6), onde com o aumento do teor de $\mathrm{Nb}$ incorporado ocorre uma brusca modificação na morfologia dos materais, tornando-se aparentemente menos cristalinos para teores elevados de $\mathrm{Nb}\left(5 \mathrm{~g}\right.$ de $\left.\mathrm{Nb}^{*}\right)$.
Tabela 1. Cristalinidades relativas dos materiais (\%).

\begin{tabular}{|l|l|l|l|l|}
\hline & Sem $\mathrm{Nb}$ & $0,5 \mathrm{Nb}$ & $1 \mathrm{Nb}$ & $5 \mathrm{Nb}$ \\
\hline Zéoliota Y & 100 & 78 & 52 & 0 \\
\hline Zeólita ZSM-5 & 100 & 93 & 91 & 24 \\
\hline
\end{tabular}

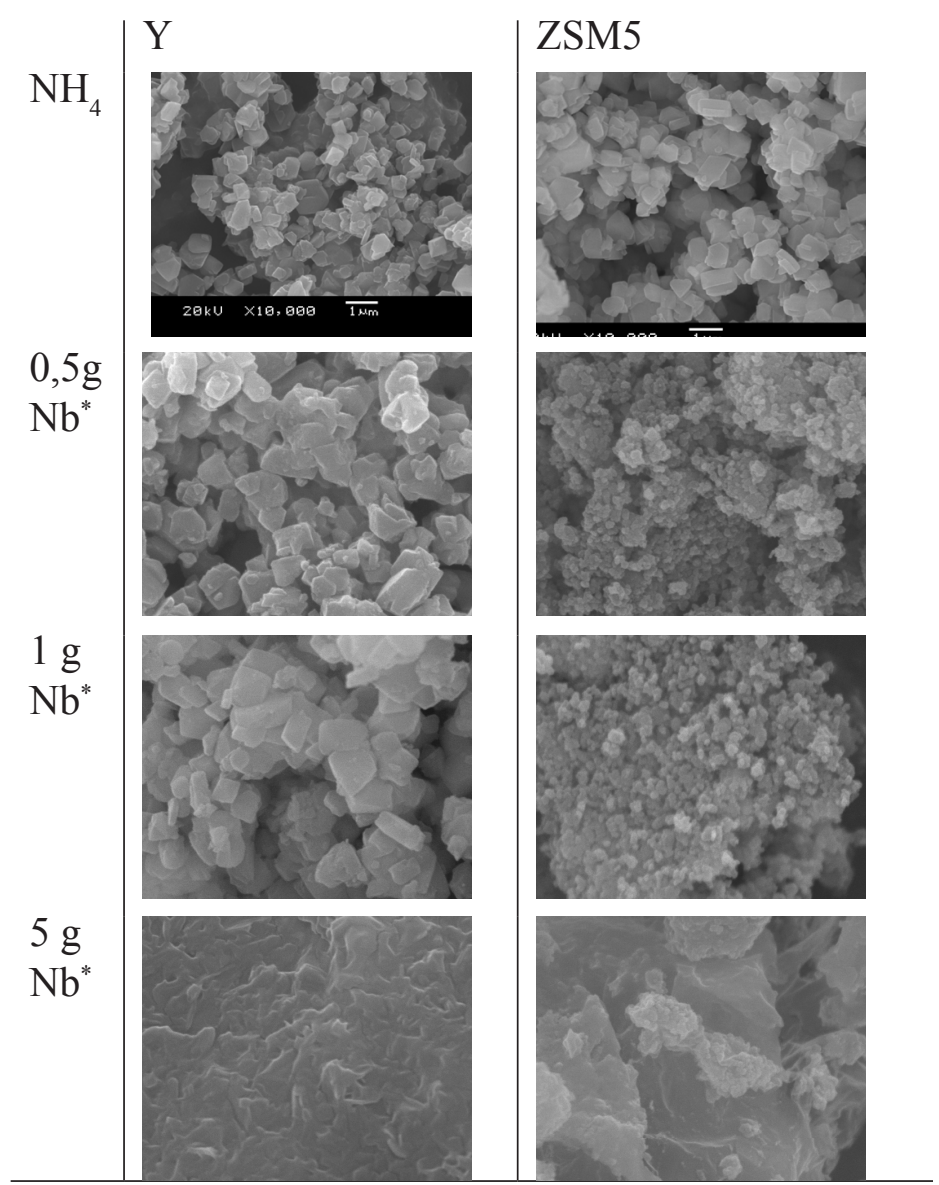

Figura 6. Micrografias dos materiais preparados.

Em todas as amostras foi realizada uma análise para determinar a área superficial específica por adsorção de Nitrogênio (BET), sendo que os resultados obtidos encontram-se na Tabela 2. Observa-se que os valores mais altos de área BET são obtidos para as zeólitas com menores quantidades de $\mathrm{Nb}$ incorporado. Com isso, verifica-se, que maiores teores de $\mathrm{Nb}$ levam à modificações nas proriedades texturais dos materiais evidenciadas pela diminuição de área superficial e de volume de poros. Esse efeito, como observado nas análises de difratometria de Raios-X, é mais pronunciado na zeólita Y. Possivelmente, isso se deve ao fato de que essa zeólita possui poros formados por 12MR, tendo uma estrutura mais aberta que a ZSM-5 (poros de 10MR), por isso possui maior área e volume de poros. Ao incorporar $\mathrm{Nb}$ (Tabela 3), provavelmente, além de uma destruição parcial da estrutura, esteja ocorrendo bloqueio dos poros 
diminuindo desta forma a acessibilidade da molécula de nitrogênio para as medidas de adsorção. A Tabela 3 apresenta a quantidade de $\mathrm{Nb}$ incorporada nas amostras e a efetividade de incorporação em relação à quantidade de $\mathrm{Nb}$ adicionada e a CTC (capacidade de troca catiônica) máxima. Observa-se que as amostras preparadas com $0,5 \mathrm{~g}$ do composto de nióbio conduziram a materiais com teores de 4,3-4,6\% de $\mathrm{Nb}$ incorporado indicando uma incorporação de aproximadamente $80 \%$ e valores abaixo da CTC máxima. Quando se empregam valores maiores de $\mathrm{Nb}$, observa-se uma maior incorporação de $\mathrm{Nb}$ nas amostras alcançando valores acima de 100\% em relação à CTC máxima. Isto significa que o excesso de $\mathrm{Nb}$ incorporado não está compensando carga, mas depositado na superfície do material.

Tabela 2. Áreas específicas e volume de poros para os materiais.

\begin{tabular}{|c|c|c|c|c|c|c|}
\hline Amostras & $\begin{array}{c}\mathrm{S}_{\mathrm{BET}} \\
\left(\mathrm{m}^{2} / \mathrm{g}\right)\end{array}$ & $\begin{array}{l}\mathrm{S}_{\mathrm{MLCRO}} \\
\left(\mathrm{m}^{2} / \mathrm{g}\right)\end{array}$ & $\begin{array}{c}\mathrm{S}_{\mathrm{EXT}} \\
\left(\mathrm{m}^{2} / \mathrm{g}\right)\end{array}$ & $\begin{array}{l}\mathrm{V}_{\text {TOTAL }} \\
\left(\mathrm{cm}^{3} / \mathrm{g}\right)\end{array}$ & $\begin{array}{l}\mathrm{V}_{\text {MICRO }} \\
\left(\mathrm{cm}^{3} / \mathrm{g}\right)\end{array}$ & $\begin{array}{c}\mathrm{V}_{\mathrm{BHJ}} \\
\left(\mathrm{cm}^{3} / \mathrm{g}\right)\end{array}$ \\
\hline HY & 503 & 453 & 50 & 0,280 & 0,234 & 0,0315 \\
\hline $\begin{array}{c}\mathrm{HY} \mathrm{Nb}^{*} \\
0,5\end{array}$ & 388 & 341 & 47 & 0,219 & 0,175 & 0,0291 \\
\hline $\begin{array}{c}\mathrm{HY} \mathrm{Nb}^{*} \\
1,0\end{array}$ & 302 & 256 & 46 & 0,173 & 0,131 & 0,0237 \\
\hline $\begin{array}{c}\mathrm{HY} \mathrm{Nb}^{*} \\
5,0\end{array}$ & 248 & 157 & 91 & 0,145 & 0,0767 & 0,0274 \\
\hline HZSM5 & 364 & 268 & 96 & 0,296 & 0,136 & 0,109 \\
\hline $\begin{array}{c}\text { HZSM5 } \\
\mathrm{Nb}^{*} 0,5\end{array}$ & 322 & 218 & 104 & 0,244 & 0,110 & 0,097 \\
\hline $\begin{array}{l}\text { HZSM5 } \\
\mathrm{Nb}^{*} 1,0\end{array}$ & 316 & 208 & 108 & 0,241 & 0,106 & 0,090 \\
\hline $\begin{array}{l}\text { HZSM5 } \\
\mathrm{Nb}^{*} 5,0\end{array}$ & 277 & 206 & 71 & 0,228 & 0,105 & 0,178 \\
\hline
\end{tabular}

Tabela 3. Análise Química dos materiais e efetividade de incorporação (\%).

\begin{tabular}{|c|c|c|c|}
\hline Amostra & $\begin{array}{c}\text { Quantidade } \\
\text { de Nb } \\
\text { incorporado } \\
\text { na amostra } \\
(\%)\end{array}$ & $\begin{array}{c}\text { Efetividade } \\
\text { da } \\
\text { incoporação } \\
\text { em relação a } \\
\text { quantidade } \\
\text { de Nb } \\
\text { adicionada } \\
(\%)\end{array}$ & $\begin{array}{c}\text { Efetividade } \\
\text { da } \\
\text { incorporação } \\
\text { em relação a } \\
\text { CTC de cada } \\
\text { material (\%)* }\end{array}$ \\
\hline HYNb0,5 & 4,6 & 83,6 & 89,5 \\
\hline HYNb1 & 7,8 & 71,2 & 152,0 \\
\hline HYNb5 & 15,5 & 28,3 & 301,7 \\
\hline HZSM5Nb0,5 & 4,3 & 78,9 & 86,7 \\
\hline HZSM5Nb1 & 6,6 & 60,3 & 132,4 \\
\hline HZSM5Nb5 & 16,8 & 30,7 & 338,0 \\
\hline
\end{tabular}

* valores acima de $100 \%$ significam que o material incorporado não está compensando carga, e sim depositado na superfície.

A eficiência catalítica dos materiais foi testada na decomposição de peróxido de hidrogênio. Os perfis de decomposição de $\mathrm{H}_{2} \mathrm{O}_{2}$ são mostrados na Figura 7.
Observa-se, de maneira geral, que os materiais com teores elevados de $\mathrm{Nb}$ apresentaram menor atividade na decomposição do peróxido de hidrogênio, isto ocorre provavelmente pela perda estrutural das zeólitas e perda de área superficial. Além disso, com maiores quantidades de $\mathrm{Nb}$ no material, pode ocorrer bloqueio dos sítios ácidos pelo metal, acarretando perdas de atividade catalítica [27]. Ao aumentar a quantidade de $\mathrm{Nb}$ incorporado na zeólita ZSM-5 de 1 para 5\%, a atividade na decomposição de $\mathrm{H}_{2} \mathrm{O}_{2}$ diminui, sendo que o tempo de decomposição passa de 15 a 30 minutos respectivamente. No caso da zeólita Y, que possui poros maiores e maior área superficial, o aumento inicial da quantidade de $\mathrm{Nb}$ incorporado (da amostra $\mathrm{HYNb0.5} \mathrm{a} \mathrm{HYNb1)} \mathrm{leva} \mathrm{a} \mathrm{um} \mathrm{acréscimo} \mathrm{na} \mathrm{atividade}$ de decomposição do $\mathrm{H}_{2} \mathrm{O}_{2}$, entretanto para quantidades elevadas de $\mathrm{Nb}$ incorporado ocorre grande perda da atividade. Esta perda de atividade ocorre supostamente devido a destruição da estrutura zeolítica evidenciada pelo difratograma de raios-X.

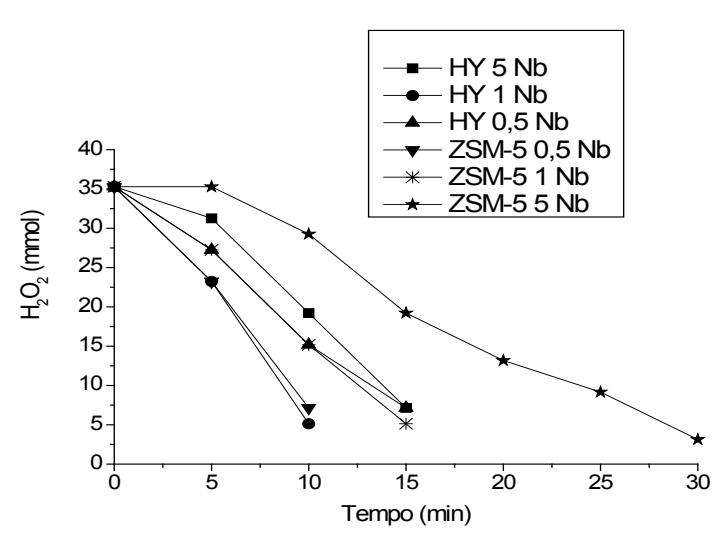

Figura 7. Decomposição de $\mathrm{H} 2 \mathrm{O} 2$.

É consenso na literatura científica [11 - 28] que a decomposição de peróxido de hidrogênio pode ocorrer via mecanismos radicalar, com formação de radicais do tipo $* \mathrm{OH}, * \mathrm{OOH}$, entre outros. No entanto, existe a possibilidade de que a decomposição ocorra via sítios vacantes como descrito por Oliveira et al 2008 [29].

$\mathrm{O}$ gráfico da Figura 8 apresenta o acompanhamento cinético mostrando a concentração de água oxigenada com o tempo de reação para a zeólita HY impregnada com $0,5 \%$ de $\mathrm{Nb}$ em três diferentes concentrações iniciais de água oxigenada. Nota-se, pela linearidade no gráfico concentração versus tempo, um comportamento teórico de uma cinética de ordem zero. Pela inclinação da reta obtém-se a constante de velocidade $(\mathrm{k})$ da reação num valor de 1,6 mol/L.min. 
A velocidade de decomposição da água oxigenada independe da sua concentração, entretanto, depende da massa de catalisador empregada como mostra o gráfico da Figura 9. Nota-se um aumento linear em k com o aumento da massa de catalisador.

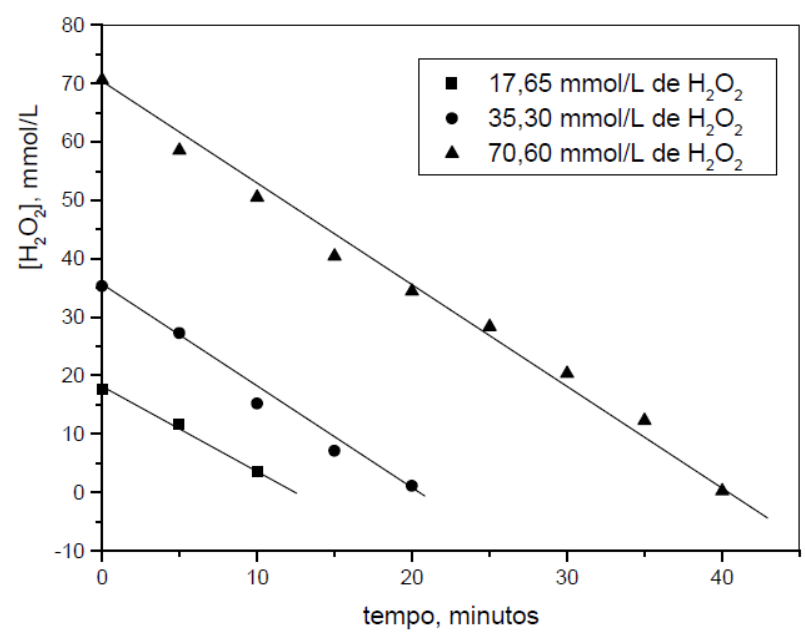

Figura 8. Concentração $\mathrm{H} 2 \mathrm{O} 2$ em função do tempo para HY0, $5 \mathrm{Nb}$.

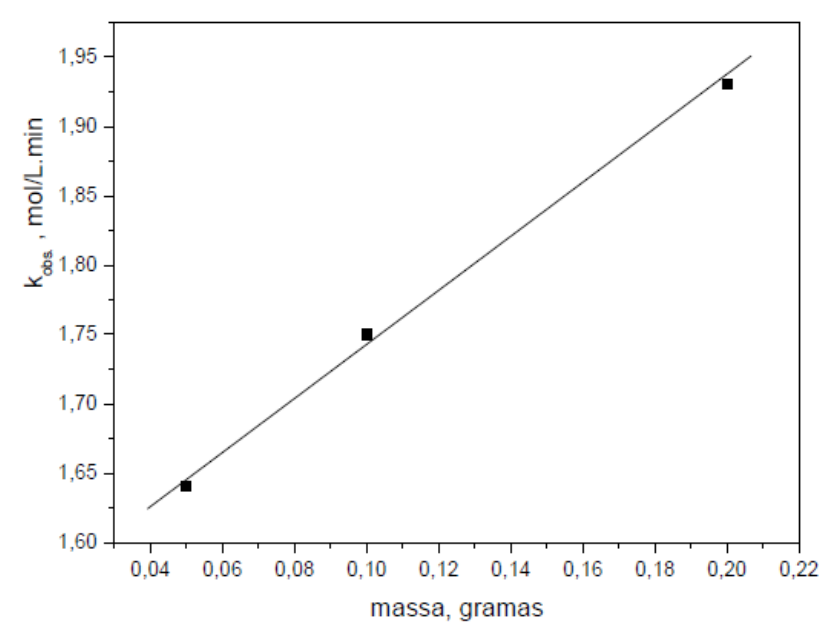

Figura 9. Constante de Velocidade (k) em função da massa do catalisador empregada.

\section{CONCLUSÕES}

A incorporação de $\mathrm{Nb}$ nas estruturas zeolíticas ZSM-5 e $\mathrm{Y}$ foi efetiva, entretanto elevados teores de $\mathrm{Nb}$ levam à obstrução dos poros e destruição parcial da estrutura cristalina. Os materiais zeolíticos impregnados com $\mathrm{Nb}$ foram ativos na decomposição do peróxido de hidrogênio, sendo que a atividade é influenciada principalmente pela estrutura do suporte zeolítico. Teores elevados de $\mathrm{Nb}$ incorporados levam a uma diminuição da atividade na decomposição do $\mathrm{H}_{2} \mathrm{O}_{2}$ possivelmente pelas modificações nas propriedades cristalinas e texturais das zeólitas.

\section{REFERÊNCIAS}

[1] M. Ziolek, Catal. Today. 78 (2003) 47.

[2] L. C. A. Oliveira, T. C. Ramalho, M. Gonçalves, F. Cereda, K. T. G. Carvalho, M. S. Nazzarro, K. Sapag, Chem. Phys. Lett. 446 (2007) 133.

[3] A. Esteves, L. C. A. Oliveira, T. C. Ramalho, M. Gonçalves, A. S. Anastácio, H. W. P. Carvalho, Catal. Commun. 10 (3) (2008) 330.

[4] T. C. Ramalho, L. C. A. Oliveira, K. T. G. Carvalho, E. F. Souza, M. Nazzarro, Mol. Phys. 107 (2009) 171.

[5] E. L. Gomes, Tese de Doutorado, Universidade Federal de São Carlos, Brasil, 2005.

[6] A. O. Florentino, M. J, Saeki, P. Cartraud, P.

Magnoux, M.Guisnet, Quím. Nova 20 (1997) 9.

[7] G.G. Pace, A. M. Rendón, G. R. Fuentes, Zeólitas: características, propiedades y aplicaciones industriale, Editorial Innovación Tecnológica Facultad de Ingeniería, UCV, Venezuela, 2000.

[8] F. Lemos; F. R. Ribeiro, M. Kern, G. Giannetto, M. Guisnet, Appl. Catal. 29 (1997) 43.

[9] http://www.dnpm.gov.br, acessada em setembro de 2009.

[10] http://www.cbmm.com.br, acessada em setembro de 2009.

[11] A. C. Silva, D. Q. L. Oliveira, L. C. A. Oliveira, A. S. Anastácio, T. C. Ramalho, J. H. Lopes, H. W. P. Carvalho, C. E. R. Torres, App. Catal. A: Gen. 357 (1) (2009) 79.

[12] L. C. A. Oliveira, M. Gonçalves,D. Q. L. Oliveira, A. L. N. Guarieiro, M. C. Pereira Quím. Nova 30 (4) (2007) 925.

[13] M. Gonçalves, M. C. Guereiro, L. C. A. Oliveira, C. L. da Rocha, Quím. Nova 31 (7) (2008) 1636.

[14] I. Nowak, M. Ziolek, Chem. Rev. 99 (12) (1999) 3603.

[15] K. Tanabe, Catal. Today 78 (2003) 65.

[16] E. Neyens, J. A. Baeyens, J. Harzad. Mater. 98(2003) 33.

[17] B. C. Larisch, J. B. Duff, Water Sci. Technol. 35 (1997) 163.

[18] W. Rempel, O. Turk, J. E. G. Sikes, J. Pulp Pap. Sci. 18 (1992) J77.

[19] E. Simpura,K Pakarinen, Tappi Environ. Conf. Proc. (1993) 865. 
[20] C. J. Lu, L. C. Fan, C. M. Lee, Water Sci. Technol. 34 (1996) 359.

[21] N. J. Rossi, Metal Finishing 95 (1997) 16.

[22] A. A. Christy, P. K. Egeberg, Talanta 51(2000) 1049 .

[23] W. J. Cooper, R. G. Zika, R. G. Petasne, J. M. C. Plane, Environ. Sci. Technol. 22 (1988) 1156.

[24] M. G. C. Baldry, J. Appl. Bacteriol. 54 (1983) 417.

[25] NFPA - National Fire Protection Agency; catalog edition, 1990.

[26] A. Maniatakou, C. Makedonas, C. A.

Mitsopoulou, C. Raptopoulou, I. Rizopoulou, A.

Terzis, A. Karaliota, Polyhedron 27 (2008) 3398.

[27] I. C. L. Barros, V. S. Braga, D. S. Pinto, J. L. de Macedo, G. N. R. Filho, J. A. Dias, S. C. L. Dias, Micropor Mesopor Mat.109 (2008) 485.

[28] W. F. Souza, I. R. Guimaraes, D. Q. Lima, C. L. T. Silva, L. C. Oliveira, Energy Fuels 23 (2009) 4426.

[29] L. C. A. Oliveira, T. C. Ramalho, E. F. Souza, M. Gonçalves, D. Q. L. Oliveira, M. C. Pereira, J. D.

Fabris, Appl. Catal. B: Environ.83 (2008) 169. 